\title{
Photoacoustic imaging depth comparison at 532-, 800-, and 1064- nm wavelengths: Monte Carlo simulation and experimental validation
}

\author{
Arunima Sharma \\ Srishti \\ Vijitha Periyasamy \\ Manojit Pramanik
}




\title{
Photoacoustic imaging depth comparison at 532-, 800-, and 1064-nm wavelengths: Monte Carlo simulation and experimental validation
}

\author{
Arunima Sharma, Srishti, Vijitha Periyasamy, and Manojit Pramanik* \\ Nanyang Technological University, School of Chemical and Biomedical Engineering, Singapore
}

\begin{abstract}
Photoacoustic imaging (PAI) provides high-resolution and high-optical-contrast imaging beyond optical diffusion limit. Further improvement in imaging depth has been achieved by using near-infrared window-I (NIR-I, 700 to $900 \mathrm{~nm}$ ) for illumination, due to lower scattering and absorption by tissues in this wavelength range. Recently, near-infrared window-II (NIR-II, 900 to $1700 \mathrm{~nm}$ ) has been explored for PAI. We studied the imaging depths in biological tissues for different illumination wavelengths in visible, NIR-I, and NIR-II regions using Monte Carlo (MC) simulations and validated with experimental results. MC simulations were done to compute fluence in tissue, absorbance in blood vessel, and in a spherical absorber (mimicking sentinel lymph node) embedded at different depths in breast tissue. Photoacoustic tomography and acoustic resolution photoacoustic microscopy experiments were conducted to validate the MC results. We demonstrate that maximum imaging depth is achieved by wavelengths in NIR-I window $(\sim 800 \mathrm{~nm})$ when the energy density deposited is same for all wavelengths. However, illumination using wavelengths around $1064 \mathrm{~nm}$ (NIR-II window) gives the maximum imaging depth when the energy density deposited is proportional to maximum permissible exposure (MPE) at corresponding wavelength. These results show that it is the higher MPE of NIR-II window that helps in increasing the PAI depth for chromophores embedded in breast tissue. $\odot$ The Authors. Published by SPIE under a Creative Commons Attribution 4.0 Unported License. Distribution or reproduction of this work in whole or in part requires full attribution of the original publication, including its DOI. [DOI: 10.1117/1.JBO.24.12.121904]
\end{abstract}

Keywords: photoacoustic imaging; photoacoustic tomography; acoustic resolution photoacoustic microscopy; near-infrared window; imaging depth; Monte Carlo simulation with embedded object.

Paper 190158SSRR received May 15, 2019; accepted for publication Jul. 18, 2019; published online Aug. 10, 2019.

\section{Introduction}

Photoacoustic imaging (PAI) is a rapidly growing biomedical imaging technique used in various clinical and preclinical applications. ${ }^{1-7}$ PAI is based on photoacoustic (PA) effect, wherein ultrasound (US) signals are generated on absorption of light by an absorber. In PAI, the target samples or tissues generate US signals after being irradiated by laser pulses. Being a hybrid imaging modality, PAI provides strong optical contrast and high US resolution, ${ }^{8}$ making it a promising tool for breast tumor imaging, ${ }^{2,9,10}$ angiogenesis monitoring, ${ }^{11,12}$ sentinel lymph node (SLN) mapping, ${ }^{13,14}$ urinary bladder imaging, ${ }^{15,16}$ monitoring of hemoglobin concentration, ${ }^{17}$ etc.

Depending upon system configuration, PAI can be broadly classified as PA computed tomography/photoacoustic tomography (PAT) and photoacoustic microscopy (PAM). In PAT, the sample is homogeneously illuminated by the laser, and the acoustic signal is collected by single or multiple ultrasound transducers (USTs) from different locations around the sample. Reconstruction algorithms are applied on the acquired signals to form images of the internal structure of the object. ${ }^{18-20}$ On the other hand, PAM generally employs confocal alignment of optical illumination and US detection, and by raster scanning of the sample, three-dimensional volumetric information of the object is obtained. Such alignment provides PAM the advantage

*Address all correspondence to Manojit Pramanik, E-mail: manojit@ ntu.edu.sg of better imaging resolution, but at a cost of relatively poor imaging depth. ${ }^{21}$

Unlike pure optical imaging modalities such as optical coherence tomography, two-photon microscopy, confocal microscopy, or Raman imaging, which have a maximum imaging depth of a few millimeters, PAI has an imaging depth up to a few centimeters. This is because PAI is based on acquisition of US signals generated by the absorbers inside the sample. The PA signal generated depends on the illumination light wavelength, the optical properties of the target sample, and propagating background medium. Light within the visible window (400 to $600 \mathrm{~nm}$ ) is generally used to image vasculature, as blood is a strong optical absorber in the visible window. ${ }^{22}$ However, surrounding tissues also absorb visible light, leading to poor imaging depth. The imaging depth can be increased by selecting suitable wavelengths for illumination, wherein scattering and absorption in background tissue are minimal. Therefore, first near-infrared window (NIR-I) (700 to $900 \mathrm{~nm}$ ) is widely used for sample illumination in PAI rather than visible (400 to $600 \mathrm{~nm}$ ) region for deep tissue imaging. ${ }^{23-26}$ Although imaging depth is increased in NIR-I, it comes with a cost of a bulky, inefficient, expensive two-stage [Ti:sapphire, dye, or optical parametric oscillator (OPO)] laser needed to generate the light in this window. Recently, a second window of near-infrared region (NIR-II, 900 to $1200 \mathrm{~nm}$ ) has been used for PAI. ${ }^{15,27-32}$ This increased interest in NIR-II imaging has been attributed to further decrease in scattering of light by tissue in this region, and to the increased maximum permissible exposure (MPE) of light 
illumination in accordance with the American National Standards Institute (ANSI) safety limit. ${ }^{33}$ However, as compared to the NIRI window, absorption of light by surrounding tissues is higher in NIR-II. The combined effect of decreased scattering, increased absorption, and increased MPE on depth of imaging in biological tissues has not been compared systematically.

In this work, we have done a comparative analysis of PAI depth in breast tissue in various optical wavelength ranges. For the first time, we have systematically compared and analyzed the penetration depth in tissue using Monte Carlo (MC) simulations and have further validated them experimentally. We compared different scenarios to account for change in imaging depth due to change in target sample, MPE at different wavelengths, and imaging modality. Since vasculature imaging and SLN imaging using external contrast agent are two common applications of PAI, environments mimicking these scenarios were considered. Different chromophores present in the two environments helped evaluate the effects of absorption coefficient $\left(\mu_{a}\right)$ of target sample on imaging depth. Further, by analyzing the results at both constant energy and MPE, relative effect on imaging depth due to change in MPE and change in tissue optical properties at different wavelengths has been examined.

MC simulations were first performed to simulate light propagation in human breast tissue with embedded targets (mimicking blood vessel or SLN) at wavelengths 532, 800, and $1064 \mathrm{~nm}$. Owing to ease of availability, 532 and $1064 \mathrm{~nm}$ are extensively used for PAI. No considerable change in optical properties is present within NIR-I; therefore, the penetration depth remains similar in the entire window. ${ }^{34,35}$ Since $800 \mathrm{~nm}$ has been used for breast vasculature imaging, ${ }^{36}$ it was considered an ideal choice for performing simulations. Another consideration for selecting the wavelengths was availability of tissue optical properties in a single publication in all the three optical windows. Therefore, for comparing penetration depths in visible, NIR-I, and NIR-II regions, wavelengths of 532, 800, and $1064 \mathrm{~nm}$ were selected, respectively.

The simulation results were then validated by performing experiments on both PAT and acoustic resolution photoacoustic microscopy (ARPAM) systems using chicken breast tissue sample and blood/ink targets. Wavelengths of 532, 740, and $1064 \mathrm{~nm}$ were employed for sample illumination in PAT. Wavelengths used for experiments were slightly different from those used for simulations, due to system restrictions. However, since changes in optical properties of blood and tissue between 740 and $800 \mathrm{~nm}$ are minimal, simulation and experimental results were expected to align. For ARPAM, samples were illuminated with 570- and 1064-nm wavelengths. The depth of imaging and the signal-to-noise ratio (SNR) were studied for all the experiments.

\section{Materials and Methods}

\subsection{Monte Carlo Simulations}

MC simulation for light propagation in multilayered tissue (MCML) with embedded objects (MCEO) was modified to compute the fluence in tissue across depth and the absorbance in objects embedded in tissue at different depths. ${ }^{37}$ The light propagation in the simulation geometry is determined by the optical properties of the medium such as $\mu_{a}$, scattering coefficient $\left(\mu_{s}\right)$, scattering anisotropy $(g)$, and refractive index $(n) .^{38}$ Three different simulation geometries were used to compare the propagation of photons at different wavelengths. Figures 1(a)1(c) illustrate the cross-sectional view of simulation geometries at $y=0$ plane, across $x z$ axis. The first simulation geometry [Fig. 1(a)] mimics human breast tissue of thickness $30 \mathrm{~cm}$. The fluence across the depth of the medium was recorded for a pencil (an infinitely narrow collimated) beam. Simulations were executed for wavelengths 532, 800, and $1064 \mathrm{~nm}$.

Figure 1(b) demonstrates the second geometry mimicking absorption of light in a blood vessel embedded in human breast tissue. A cylinder of 1-mm diameter and infinite length was placed at different depths in the tissue medium, which was subjected to pencil beam illumination. Absorbance in cylinder was computed at different wavelengths by varying optical properties of the medium and the cylinder to match the properties of human breast tissue and blood, respectively. ${ }^{39,40}$ Distance between the tissue surface and the cylinder was increased to compute the absorption of light by the blood vessel at different depths.

MCEO was further performed on the geometry as illustrated in Fig. 1(c). Here, absorbance was calculated in a sphere of diameter $1 \mathrm{~cm}$ placed at different depths in tissue. The $\mu_{a}$ of the sphere was constant $\left(=1 \mathrm{~cm}^{-1}\right)$ across all wavelengths, while its $\mu_{s}$ was equal to the $\mu_{s}$ of surrounding tissue. Such geometry mimics imaging of organs such as urinary bladder or SLN, which generally require injecting contrast agent such as methylene blue, indocyanine green, gold nanoparticles, and black ink, during imaging. ${ }^{41}$ The sphere, therefore, mimics the organ filled with contrast agent. Similar to the previous case, the position of the sphere and the properties of the sphere and the tissue were modified to get absorption at different depths and at different wavelengths, respectively. Table 1 gives the optical properties of medium, cylinder, and sphere, used for simulations.

$10^{8}$ photons were launched for each simulation to ensure high computational accuracy. The cylinder and sphere were embedded at depths 1 to $6 \mathrm{~cm}$ from the tissue surface in steps of $1 \mathrm{~cm}$. All simulations were performed on a desktop computer with an Intel Xeon 3.7 GHz, 64-bit processor, and 16-GB RAM running windows 10 operating system.

\subsection{Acoustic Resolution Photoacoustic Microscopy Imaging System Configuration}

ARPAM system was used for comparing the images obtained using laser illumination of 570 and $1064 \mathrm{~nm}$. A nanosecond diode-pumped solid-state Nd:YAG laser (INNOSLAB, Edgewave, Wurselen, Germany) generated laser pulses of wavelengths 532 and $1064 \mathrm{~nm}$ at a pulse repetition rate (PRR) of $5000 \mathrm{~Hz}$. A harmonic beam splitter (HBSY11) reflected a 1064-nm beam toward the 1064-nm ARPAM system and the transmitted 532-nm beam was allowed to pass through a dye laser (Credo-DYE-N, Sirah dye laser, Spectra Physics, Santa Clara, California) toward the 570-nm ARPAM system. More detailed system descriptions (and performance) of the 570$\mathrm{nm}$ ARPAM system ${ }^{42}$ and the 1064-nm ARPAM system ${ }^{15}$ have been reported earlier. The PA signal generated by the sample after irradiation with the $570-\mathrm{nm}$ beam was collected by a 50-MHz UST (V214-BB-RM, Olympus NDT, Waltham, Massachusetts) housed in the center of an optical condenser. Before acquisition, the PA signal was focused with an acoustic lens (LC 4573, Thorlabs) having a radius of curvature of $4.6 \mathrm{~mm}$ and a diameter of $6 \mathrm{~mm}$, attached to the UST. For the 1064-nm ARPAM system, a 30-MHz UST attached to a diverging lens (LC1975-C, Thorlabs) of diameter $6 \mathrm{~mm}$ and radius of curvature 

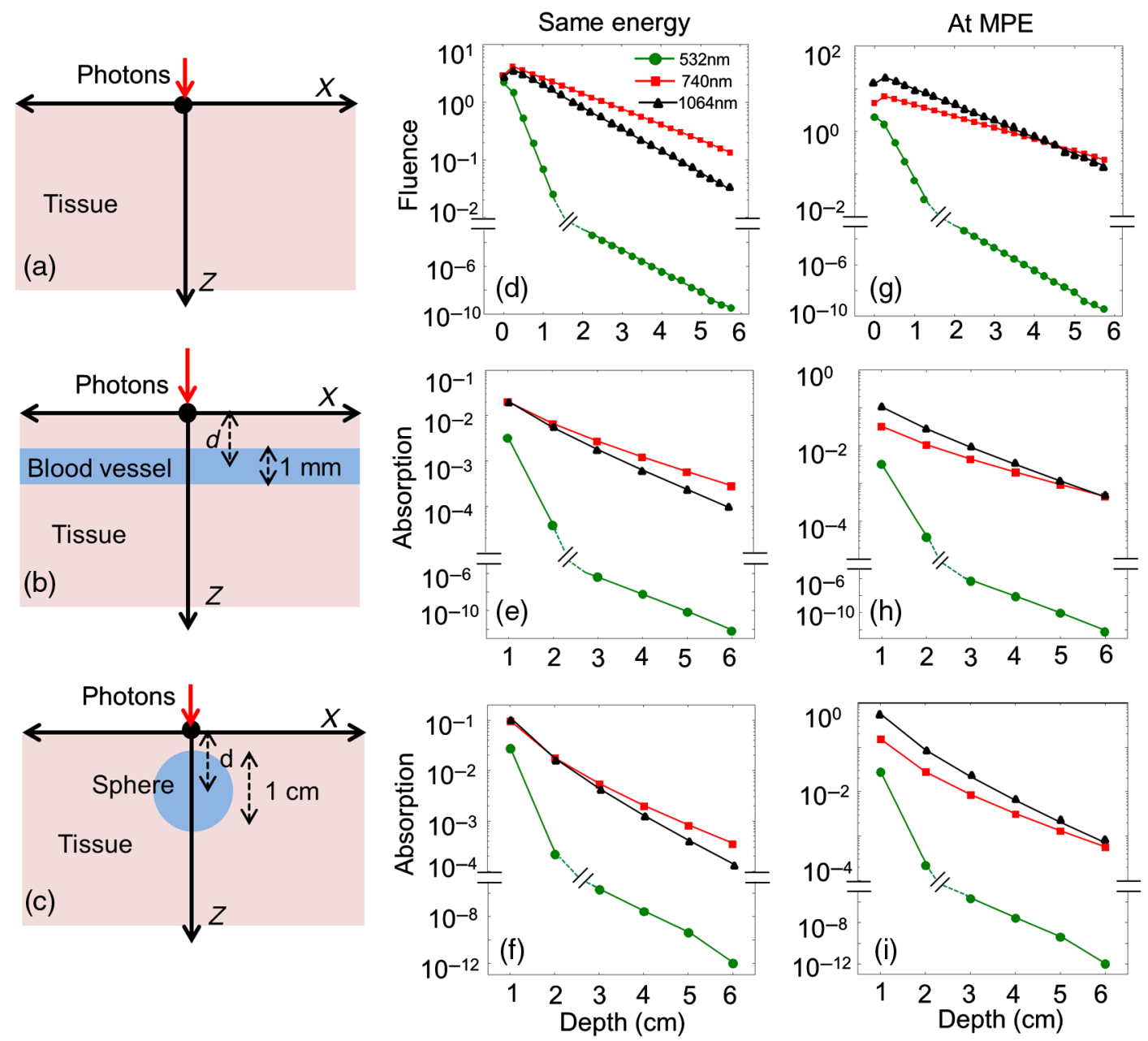

Fig. 1 (a)-(c) Cross-sectional view of simulation geometry at $y=0$ during MC simulations for (a) uniform tissue medium, (b) tissue with cylinder mimicking blood properties placed with center at depth $d$ from surface, and (c) tissue with sphere mimicking constant absorber placed with center at depth $d$ from surface. (d)-(f) Results of MC simulation corresponding to geometries (a)-(c) showing (d) fluences across depth for different wavelengths, (e) absorbance in cylinder, and (f) absorbance in sphere. (g)-(i) Results obtained for (d)-(f) after multiplication with factors proportional to MPE. Symbols for (d)-(i) are same as shown in (d).

Table 1 Optical properties of surrounding medium, cylinder, and sphere, used for MC simulations. Refractive index $(n)$ was 1.33 for all the mediums.

\begin{tabular}{|c|c|c|c|c|c|c|c|c|c|}
\hline \multirow{2}{*}{$\begin{array}{l}\text { Layer } \\
\text { Wavelength }(\mathrm{nm})\end{array}$} & \multicolumn{3}{|c|}{ Medium (human breast tissue) ${ }^{39}$} & \multicolumn{3}{|c|}{ Cylinder (blood vessel) ${ }^{40}$} & \multicolumn{3}{|c|}{ Sphere (constant absorber) } \\
\hline & 532 & 800 & 1064 & 532 & 800 & 1064 & 532 & 800 & 1064 \\
\hline$\mu_{a}\left(\mathrm{~cm}^{-1}\right)$ & 0.45 & 0.011 & 0.033 & 224.8 & 3.8 & 4.5 & 1 & 1 & 1 \\
\hline$\mu_{s}\left(\mathrm{~cm}^{-1}\right)$ & 120 & 116 & 77 & 727.8 & 767.3 & 592.6 & 120 & 116 & 77 \\
\hline$g$ & 0.9 & 0.9 & 0.9 & 0.9687 & 0.9833 & 0.9799 & 0.9 & 0.9 & 0.9 \\
\hline
\end{tabular}

of $12.4 \mathrm{~mm}$ was used to acquire the generated PA signal. The UST was positioned at the center of the optical condenser to achieve confocal alignment of optical and acoustic foci.

In both the ARPAM systems, the scanning setup was mounted on a three-axis motorized stage (PLS 85 for $x$ and $y$ axes, VT 80 for $z$ axis, PI-Physik Instrumente, Karlsruhe, Germany) which was controlled by a three-axis controller (SMC corvus eco, PI miCos). The scanning head was immersed in a water tank $(13 \times 30 \mathrm{~cm})$ having an imaging window with diameter of $10 \mathrm{~cm}$ sealed with polyethylene membrane.

\subsection{Photoacoustic Tomography Imaging System Configuration}

The system configuration (and performance) for PAT ${ }^{43}$ system has been described in detail earlier. A Q-switched Nd:YAG laser 
delivered laser pulses of wavelengths 532 and $1064 \mathrm{~nm}$ at a PRR of $10 \mathrm{~Hz}$. These pulses were split using a harmonic beam splitter (HBSY11) before being guided toward the imaging area. For generating pulses of wavelength $740 \mathrm{~nm}$, the beam from $\mathrm{Nd}$ : YAG laser was pumped into a tunable OPO (Continuum, Surelite OPO) laser. The desired wavelength was guided to the sample using three right-angled prisms (PS911, Thorlabs). The setup was arranged such that the sample was irradiated homogeneously by laser pulses from above. An optical diffuser was used to homogenize the beam.

The PA signal generated by the sample was collected using a 5-MHz single-element UST (V309-SU, Olympus NDT) having a 13-mm diameter active area and a $70 \%$ nominal bandwidth. UST was connected to a stepper motor (Lin Engineering, Silverpak 23C) which rotated it around continuously to collect signal from different angles. Both the sample and the UST were placed in a water tank to enable better coupling of US signal.

\subsection{Sample Preparation}

Two transparent, low-density polyethylene (LDPE) tubes having an inner diameter of $0.59 \mathrm{~mm}$ were filled with black ink and fresh blood, respectively. Blood was chosen owing to its vast use as an endogenous light absorber in the body. Black ink, due to its high coefficient of absorption across the visible and NIR spectrum, mimics the properties of any exogenous contrast agent used during PAI.

For ARPAM, the tubes were placed under the imaging window of the water tank, while for PAT, they were directly placed inside the tank. Slices of different thicknesses of commercially available chicken breast tissue were stacked on the LDPE tubes to illustrate imaging at different depths. ARPAM data were acquired by raster scanning over the tubes in an area of $8 \mathrm{~mm} \times 10 \mathrm{~mm}$. Maximum amplitude projection (MAP) of the acquired data was computed using MATLAB (MathWorks, Natick, Massachusetts). PAT data were acquired by rotating the UST around the sample at a speed of $1.5 \mathrm{deg} / \mathrm{s}$. The collected data were averaged into 240 A-lines to compensate for pulse-topulse variation of laser power. A simple delay-and-sum algorithm ${ }^{44}$ was implemented for data reconstruction.

United States Air Force 1951 test target (R1DS1P, Thorlabs) was further imaged to validate the depth of imaging in ARPAM systems. The target was placed below the imaging window, and slices of chicken breast were stacked on it. The scanning head was raster scanned in an area of $20 \mathrm{~mm} \times 20 \mathrm{~mm}$ across the test target. Transparent US gel was used as a coupling medium between the sample and the imaging window. MAP of the acquired data was computed to form the final image.

\subsection{Laser Power}

Laser energy density on the surface of biological tissues is governed by ANSI safety limits. ${ }^{33}$ The safety limit depends on incident light wavelength and time of exposure on the sample. To analyze the effect on imaging depth due to change in optical properties of tissue (which is dependent on the wavelength of illumination) and change in MPE of light independently, experiments were conducted in two ways. Initially, imaging depth was compared using constant laser fluence across all wavelengths. Later, the fluence was changed proportionally to the MPE at that wavelength.

For ARPAM systems used here, the allowable per-pulse energy density for the 570 and $1064 \mathrm{~nm}$ are $1^{42}$ and
Table 2 Per-pulse fluence and multiplication factors for different wavelengths.

\begin{tabular}{lccc}
$\begin{array}{l}\text { Wavelength } \\
(\mathrm{nm})\end{array}$ & $\begin{array}{c}\mathrm{MPE}^{\mathrm{a}} / \text { pulse } \\
\left(\mathrm{mJ} \mathrm{cm}^{-2}\right)^{33}\end{array}$ & $\begin{array}{c}\text { Energy density/pulse } \\
\left(\mathrm{mJ} \mathrm{cm}^{-2}\right)(\mathrm{PAT} \\
\text { experiments })\end{array}$ & $\begin{array}{c}\text { Multiplication } \\
\text { factor }(k) \\
(\text { simulation })\end{array}$ \\
\hline 532 & 20 & 4.2 & 1 \\
740 & 24 & 5.1 & $\mathrm{~N} / \mathrm{A}$ \\
800 & 32 & $\mathrm{~N} / \mathrm{A}$ & 1.6 \\
1064 & 100 & 21.4 & 5 \\
\hline
\end{tabular}

${ }^{\mathrm{a} M P E}$ for more than $10 \mathrm{~s}$ of skin exposure for $10-\mathrm{Hz}$ laser.

$2.96 \mathrm{~mJ} / \mathrm{cm}^{2},{ }^{15}$ respectively. To analyze images at constant laser power, experiments were conducted at pulse energy of $0.30 \mathrm{~mJ} / \mathrm{cm}^{2}$ for both the wavelengths. Later, pulse energy of the 1064-nm system was increased to $1 \mathrm{~mJ} / \mathrm{cm}^{2}$ and the acquired images were compared with the images obtained previously using the 570-nm system, to compare the results when energy is proportional to MPE.

Owing to the change in exposure time, the allowable perpulse energy density was different for PAT. In this case, initial experiments were conducted by maintaining a constant energy density of $5.1 \mathrm{~mJ} / \mathrm{cm}^{2}$ across all wavelengths. Later, pulse energy of the 532- and 1064-nm pulses was altered such that it became proportional to MPE at respective wavelengths. Table 2 provides the MPE and the per-pulse energy densities for different wavelengths used during PAT experiments.

For MCEO results, MPE was calculated for exposure duration between $10 \mathrm{~s}$ and $3 \times 10^{4} \mathrm{~s}$. Simulations were performed to give values of absorbance and fluence at different wavelengths and different depths. These values were multiplied with a multiplication factor $(k)$ proportional to MPE, to give an estimate of relative absorbance at MPE. Values of MPE and $k$ used during simulations are provided in Table 2.

\section{Results}

Figures 1(d)-1(i) show the results of MC simulations when tissue [Figs. 1(d) and $1(\mathrm{~g})$ ] and tissue with embedded objects [Figs. 1(e), 1(f), 1(h), and 1(i)] were illuminated by a pencil beam at different wavelengths. Fluence across depth in human breast tissue for wavelengths 532, 800, and $1064 \mathrm{~nm}$ has been shown in Fig. 1(d). Figures 1(e) and 1(f) show absorbance in cylinder and sphere, respectively, when they were embedded at different depths in breast tissue. Corresponding results of Figs. 1(d), 1(e), and 1(f) at MPE are shown in Figs. 1(g), 1(h), and 1(i), respectively. The results obtained show a drastic decrease in tissue fluence with an increase in depth for illumination with wavelength of $532 \mathrm{~nm}$. The decrease in fluence resulted in a corresponding decrease in absorbance in both cylinder and sphere. This decrease can be attributed to the high $\mu_{a}$ and high $\mu_{s}$ of breast tissue in visible region compared to NIR window.

The change in absorption with depth is much lower in NIR region, as compared to visible region. It can be observed in Figs. 1(d)-1(f) that there is a higher decrease in absorption at 1064-nm illumination than at 800-nm illumination when the energy deposited for both the wavelengths is same. Although the $\mu_{s}$ decreases with increase in wavelength, the $\mu_{a}$ in breast 
tissue is higher at $1064 \mathrm{~nm}$ than at $800 \mathrm{~nm}$. This increase in $\mu_{a}$ results in a decrease in the amount of light reaching higher depths. Conversely, it was observed that when the tissue was subjected to exposure by all three wavelengths at MPE, fluence was highest for $1064 \mathrm{~nm}$ till an approximate depth of $4.5 \mathrm{~cm}$, after which fluence for $1064 \mathrm{~nm}$ reduces compared to $800 \mathrm{~nm}$. The absorbance of $1064 \mathrm{~nm}$ is higher than the absorbance of $800 \mathrm{~nm}$, in both the cylinder and the sphere at MPE till a depth of $6 \mathrm{~cm}$. Between 4.5 and $6 \mathrm{~cm}$, the fluence in tissue for the 1064-nm illumination reduces with depth compared to the $800 \mathrm{~nm}$, while the absorbance in the objects for $1064 \mathrm{~nm}$ remain higher than $800 \mathrm{~nm}$. This observation can be attributed to the different properties of embedded objects at different wavelengths. Higher $\mu_{s}$ of embedded objects at $800 \mathrm{~nm}$ (compared to $1064 \mathrm{~nm}$ ) might result in decreased absorbance of light in some parts of sphere, even though fluence in the given $x y$ plane is larger.

It can be concluded from simulations that the fluence decreases by almost $96 \%$ per $\mathrm{cm}$ in visible $(532 \mathrm{~nm})$ region, while the average decrease in NIR-I $(800 \mathrm{~nm})$ is $\sim 30 \% / \mathrm{cm}$ and the average decrease in NIR-II $(1064 \mathrm{~nm})$ is $45 \%$ per $\mathrm{cm}$. Thus, the absorbance changes by a factor of $\sim 100 / \mathrm{cm}$ in visible region and by a factor $<10$ in NIR region. Further, till $\sim 6 \mathrm{~cm}$ depth, NIR-I has higher absorbance in embedded object when laser power is same, while NIR-II has higher absorbance in embedded objects when laser power is proportional to MPE.

Experiments were conducted to validate the simulation results. Figure 2 shows the results obtained when LDPE tubes filled with black ink and blood were subjected to ARPAM experiments. Figures 2(a)-2(e) correspond to images obtained
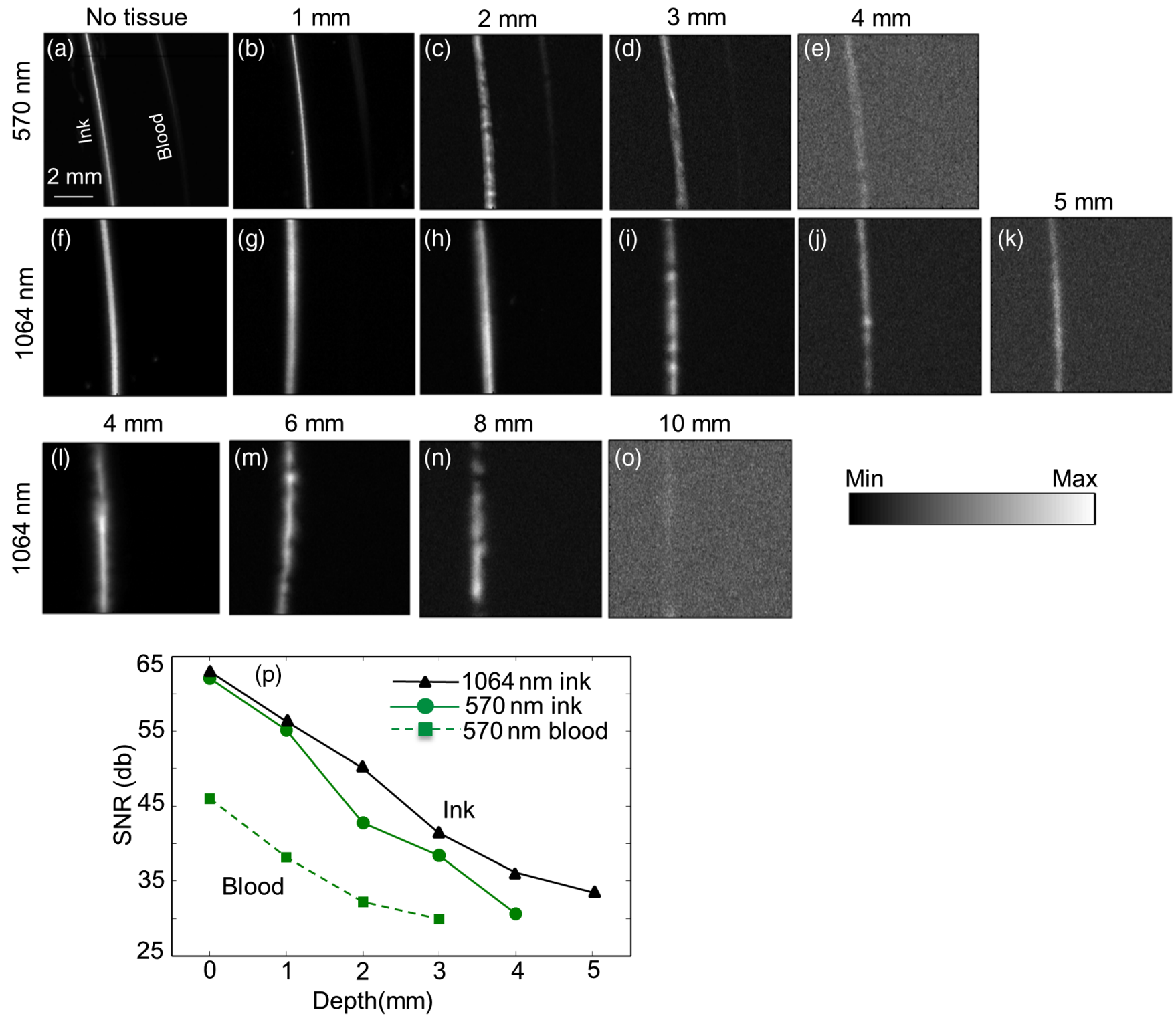

Fig. 2 Comparison of images obtained using (a)-(e) 570-nm ARPAM and (f)-(o) 1064-nm ARPAM system at (a)-(k) same fluence and (a)-(e), (l)-(o) fluence proportional to MPE. Left and right tubes were filled with black ink and blood, respectively. Scale bar is same for all images as given in (a). Numbers on top of images indicate the thickness of tissue placed on the tubes. (p) The SNR for (a)-(k). Solid lines indicate ink and dashed lines indicate blood. 
using the 570-nm ARPAM system, while Figs. 2(f)-2(o) correspond to images obtained using the 1064-nm ARPAM system. In the images, the tube on the left was filled with ink, while that on the right [only visible in Figs. 2(a)-2(c)] was filled with blood. Laser fluence per pulse was maintained at $0.30 \mathrm{~mJ} / \mathrm{cm}^{2}$ for Figs. 2(a)-2(k) and at $1 \mathrm{~mJ} / \mathrm{cm}^{2}$ for Figs. 2(l)-2(o).

At same fluence, the tube containing ink was visible till a depth of $4 \mathrm{~mm}$ in the 570-nm ARPAM system and $5 \mathrm{~mm}$ in the 1064-nm ARPAM system. Further, on increasing the surface fluence of the 1064-nm ARPAM system such that the surface fluence for both the wavelengths were proportional to MPE at respective wavelength, the imaging depth increased to almost $10 \mathrm{~mm}$. This corresponds to more than $100 \%$ increase in imaging depth between 570 and 1064 nm ARPAM systems for black ink. However, $570 \mathrm{~nm}$ showed a much stronger blood absorbance as compared to $1064 \mathrm{~nm}$. LDPE tube filled with blood was visible till a depth of $3 \mathrm{~mm}$ in the 570-nm system, while it was not at all visible in the 1064-nm system. Visibility of blood tubes in these images is poor due to a high relative contrast of black ink. Tubes were clearly visible in Figs. 2(a)-2(c) on adjusting the color bar (images not shown). For quantification of results, SNR at different depths was computed as

$\operatorname{SNR}(\mathrm{dB})=10 \times \log V / N$,

where $V$ is the mean of PA signal (above a threshold) from an area where sample was placed and $N$ is the standard deviation of the background noise.

Figure 2(p) shows SNR for ink (solid lines) and blood (dotted lines) for the 1064- (black triangles) and 570-nm (green circles and squares) systems corresponding to images in Figs. 2(a)2(k). The average change in SNR of black ink was more $(8 \mathrm{~dB} / \mathrm{mm})$ for the $570-\mathrm{nm}$ system, as compared to the $1064-\mathrm{nm}$ system $(6 \mathrm{~dB} / \mathrm{mm})$. SNR of blood was $25-30 \%$ less than the SNR of ink for the $570 \mathrm{~nm}$ ARPAM system. Owing to low SNR, blood was not visible beyond a depth of $3 \mathrm{~mm}$.

Experiments were further conducted on test targets to assess the images obtained at different imaging depths. Figures 3(a), 3(b) and 3(c)-3(e) show images obtained in the 570- and 1064-nm systems, respectively. Chicken tissue of thicknesses 0,3 , and $8 \mathrm{~mm}$ were stacked on targets in Figs. 3(a), 3(c); 3(b), 3(d); and 3(e), respectively. It was observed that when no tissue was placed on the sample, the images obtained using the 570-nm system showed better image quality, as compared to images obtained on using the 1064-nm system. This may be attributed to the different USTs used in the two imaging systems. Since the imaging depth of the 1064-nm system is higher, a transducer with lower central frequency was used for signal detection. This resulted in a decrease in system resolution. ${ }^{45}$ It can also be observed from Figs. 3(c)-3(e) that an increase in tissue thickness results in an increase in the image artifacts. Presence of chicken tissue results in refraction of PA signal at multiple surfaces. Owing to the nonuniformity of tissue, refraction at different points across the image is different, resulting in slight changes in acoustic focus and acoustic impedance. Therefore, some parts of the image appear out of focus. With an increase in tissue thickness, slight degradation in image quality was observed. However, the structures of test targets were distinct and intact. This confirms that imaging of bigger structures such as lymph node and urinary bladder can be accurately performed at higher depths using the 1064-nm ARPAM system; however, smaller structures with low absorbance might be difficult to discern at such depths.

Following this, experiments at wavelengths of 532, 740, and $1064 \mathrm{~nm}$ were conducted using PAT system. The images of LDPE tubes filled with blood and black ink are shown in Figs. 4 and 5. The left tube in the image was filled with black ink, while the right one was filled with blood. Figure 4 shows images acquired when the tubes were irradiated with constant surface fluence for all wavelengths. SNR values, computed in the same way as explained earlier, for blood (dotted lines) and black ink (solid lines) at different depths, for all three wavelengths are shown in Fig. 4(o). The graph shows that PA signal from blood was maximum at $532 \mathrm{~nm}$ in the absence of chicken tissue. This is expected due to high $\mu_{a}$ of blood at $532 \mathrm{~nm}$. An increase in depth of chicken tissue resulted in a decrease in SNR
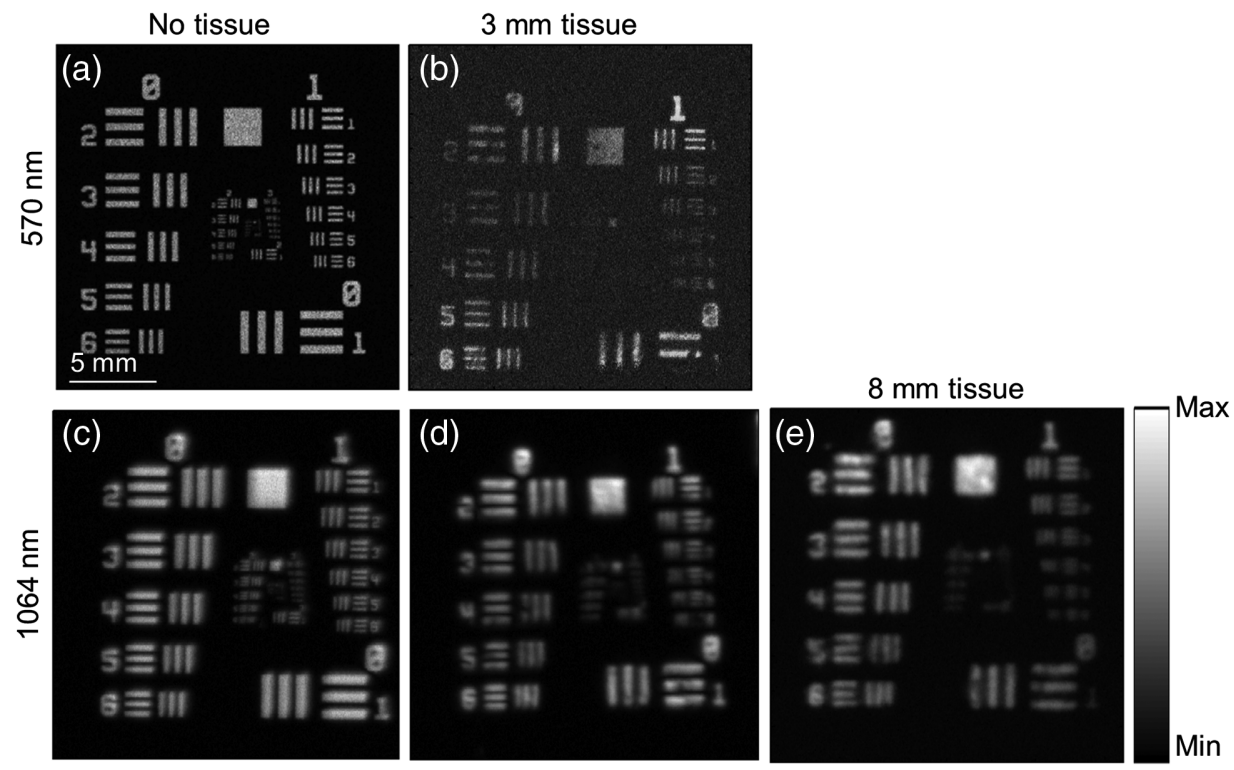

Fig. 3 Images of test targets acquired using (a) and (b) 570-nm ARPAM system and (c)-(e) 1064-nm ARPAM system when (a) and (c) no tissue; (b) and (d) 3-mm tissue; and (e) 8-mm tissue were stacked on the targets. Scale bar is same for all images as shown in (a). 

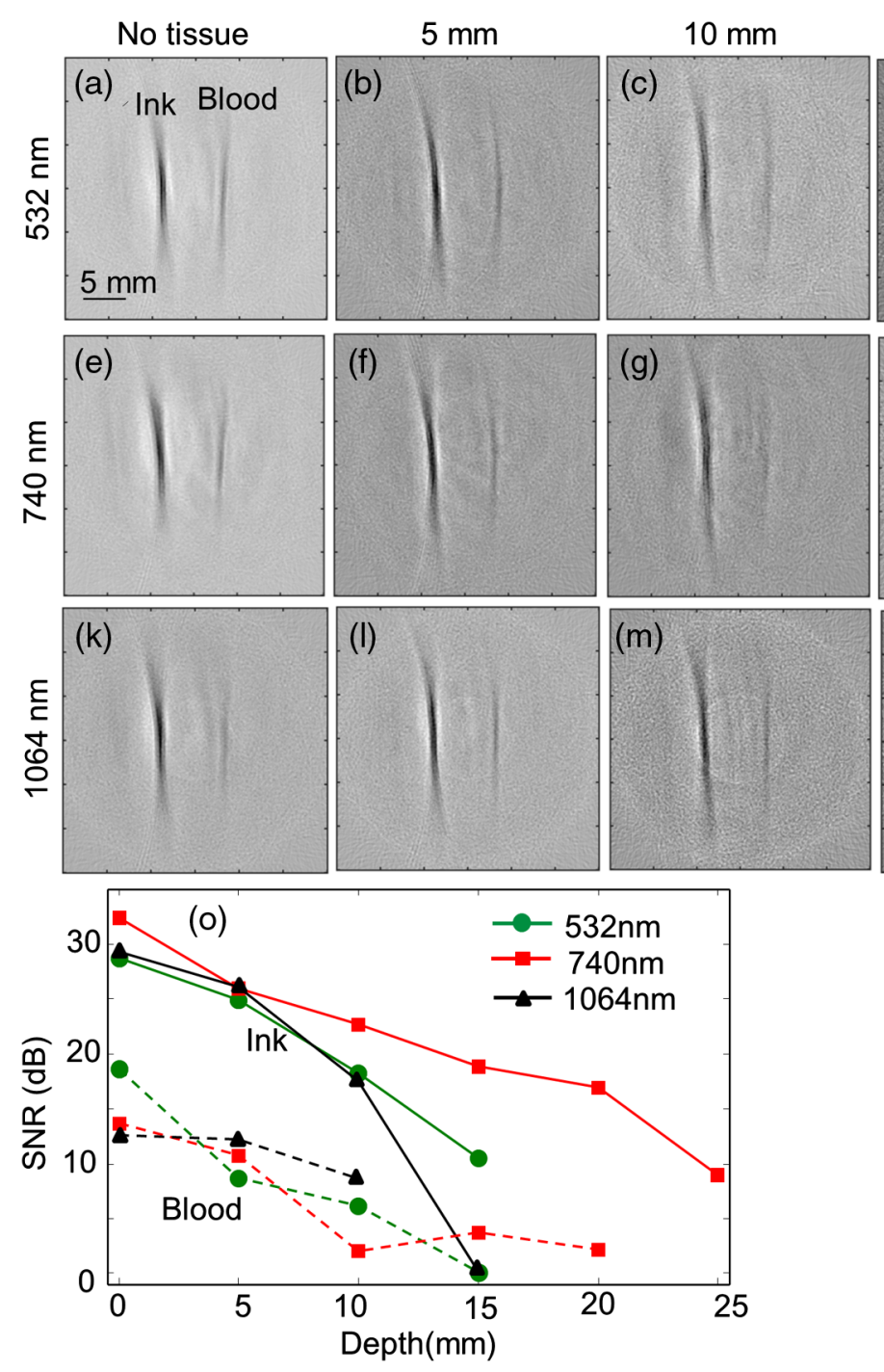

Fig. 4 Comparison of images acquired using PAT at (a)-(d) 532-nm, (e)-(j) 740-nm, and (k)-(n) 1064-nm laser irradiation with same surface fluence. Scale bar is same for all images as shown in (a). Left and right tubes were filled with black ink and blood, respectively. Numbers on top of the images indicate the thickness of tissue placed on the tubes. (o) SNR for black ink (solid lines) and blood (dotted lines) at different depths for different wavelengths.

for all three wavelengths. The decrease in SNR per unit length was similar for 532 and $1064 \mathrm{~nm}$ and was slightly lower for $740 \mathrm{~nm}$. No particular trend in SNR variation was observed. For 532 and $1064 \mathrm{~nm}$, the LDPE tubes containing black ink were discernible only till imaging depth of $15 \mathrm{~mm}$, while for $740 \mathrm{~nm}$, the tube was visible till $25 \mathrm{~mm}$. Thus, the experimental results validated the simulation results, which suggested that at constant fluence, absorbance in constant absorber at higher depths will be maximum for NIR-I region.

Figures 5(a)-5(1) show the images acquired for LDPE tubes when the surface fluence at different wavelengths was as given in Table 2. Corresponding SNR values for blood and ink at all three wavelengths are shown in Fig. 5(m). Owing to a relatively low MPE in visible region, the SNR for blood and ink for $532 \mathrm{~nm}$ was lower than that observed in the previous case. Black ink was still visible to a depth of $15 \mathrm{~mm}$, but image quality degraded due to reduced SNR. However, due to much higher MPE of NIR-II, the SNR values at $1064 \mathrm{~nm}$ were at least 30\% higher than the SNR values at $740 \mathrm{~nm}$ for imaging depths between 10 and $25 \mathrm{~mm}$. The imaging depth for $1064 \mathrm{~nm}$ increased to $35 \mathrm{~mm}$ at this surface fluence. For depths beyond $25 \mathrm{~mm}$, the LDPE tubes were not discernible with $740 \mathrm{~nm}$. Imaging of tissue $<10 \mathrm{~mm}$ was not performed with $1064 \mathrm{~nm}$ in this case, as such high power could have resulted in boiling of black ink, thus damaging the tube.

As can be seen in Table 2, MPE for all wavelengths was higher than the surface fluence used for the experiments. The laser power was limited due to the use of OPO, which was used for generating 740-nm pulses. Energy of Nd:YAG laser could further be increased to achieve an imaging depth of $45 \mathrm{~mm}$ at 1064-nm wavelength, as shown in Figs. 5(n) and 5(o). For all the images obtained using PAT, other image characteristics, such as resolution and spatial accuracy, remained same irrespective of the illumination wavelength used.

\section{Discussion and Conclusion}

In this work, imaging depths in breast tissue for different wavelengths have been compared using both MC simulations and 


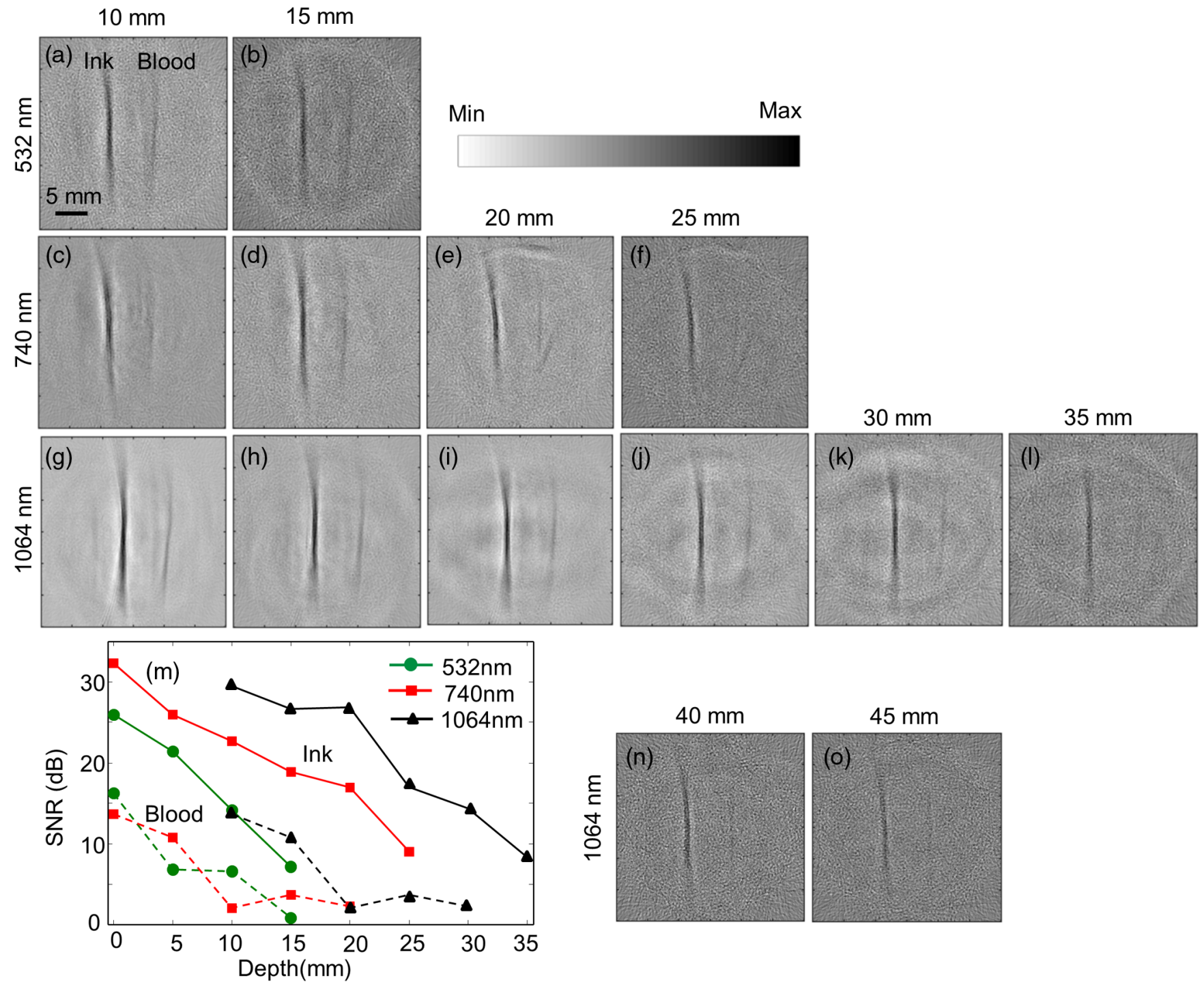

Fig. 5 Comparison of images acquired using PAT at (a) and (b) 532-nm, (c)-(f) 740-nm, and (g)-(l) 1064$\mathrm{nm}$ laser irradiation with surface fluence proportional to MPE. Scale bar is same for all images as shown in (a). Left and right tubes were filled with black ink and blood, respectively. $(\mathrm{m})$ SNR for black ink (solid lines) and blood (dotted lines) at different depths for different wavelengths. (n) and (o) Images acquired at 1064-nm illumination when surface fluence was increased to maximum possible value. Numbers on top of the images indicate the thickness of tissue placed on the tubes.

experiments. Two environments, one mimicking a blood vessel inside human breast tissue and another mimicking imaging of SLN following injection of a contrast agent, were considered for simulations. Tissue properties corresponding to three wavelengths, one each in visible, NIR-I, and NIR-II regions were used. Simulation results showed a severe decrease in laser fluence for visible region $(532 \mathrm{~nm})$ with an increase in depth of breast tissue. It was also observed that the decrease in fluence with depth was more for NIR-II (1064 nm) as compared to NIRI $(800 \mathrm{~nm})$, when both were irradiated with same number of photons. At MPE, the fluence for $1064 \mathrm{~nm}$ was higher till an approximate depth of $4.5 \mathrm{~cm}$, following which fluence of 800 and $1064 \mathrm{~nm}$ became equal and then fluence at $800 \mathrm{~nm}$ became larger. MCML simulations may further be theoretically convolved to compare penetration depth for different beam profiles; however, the trend in imaging depth for different wavelengths is expected to remain similar for all profiles.
Following simulations, experiments were conducted on both ARPAM and PAT systems. ARPAM experiments showed an increase in imaging depth for $1064 \mathrm{~nm}$, as compared to $570 \mathrm{~nm}$. The increment in depth of chicken breast was from 4 to $5 \mathrm{~mm}$, when both the systems had same fluence, and from 4 to $10 \mathrm{~mm}$, when fluence was proportional to MPE. To validate that the imaging depth of the 570-nm system was low due to the wavelength and not due to the UST used, experiments were conducted by irradiating the sample at $570 \mathrm{~nm}$ but by using a 30$\mathrm{MHz}$ UST instead of a 50-MHz UST (results not shown). The imaging depth using this setup was similar to what was observed in a conventional 570-nm ARPAM system, as described above. Thus, confirming that the comparatively lower imaging depth was due to high $\mu_{a}$ and $\mu_{s}$ in tissue at $570 \mathrm{~nm}$.

PAT experimental results validated the simulation results that the decrease in SNR with imaging depth was more for NIR-II $(1064 \mathrm{~nm})$ as compared to NIR-I $(740 \mathrm{~nm})$ when samples were 
irradiated with constant surface fluence. This resulted in higher achievable imaging depth in chicken breast tissue for $740 \mathrm{~nm}$. Nevertheless, analogous to simulation results, the SNR for NIRII was higher than the SNR for NIR-I, when pulse energy in both cases was proportional to MPE at that wavelength. Therefore, in this case, the imaging depth was higher for NIR-II, as compared to NIR-I. For our experiments, imaging depths in chromophores embedded in chicken breast were 15,25 , and $15 \mathrm{~mm}$, respectively, for wavelengths 532, 740, and $1064 \mathrm{~nm}$ when irradiated by same surface fluence. The imaging depths changed to 15,25 , and $35 \mathrm{~mm}$, respectively, for 532-, 740-, and 1064-nm wavelengths, when surface fluence was proportional to MPE. Although the simulation and experimental results follow similar trend, slight differences could still be observed. This is due to the differences in optical properties of human breast tissues used in simulations versus the chicken breast tissues used in the experiments. Further variation in penetration depth for all wavelengths may be observed on changing the type of tissue (e.g., muscles and liver). In addition, variation can be observed on using excitation wavelengths different from the ones that have been used in this study, even though they might be within the same optical window. One needs to use appropriate simulation or experiments to find out the imaging depth for different combination of tissue types or wavelengths.

Our results show that the imaging depth in breast tissue for $1064 \mathrm{~nm}$ was higher than $740 \mathrm{~nm}$ due to its higher MPE. Compared to visible region, NIR regions have higher imaging depths, owing to the decreased scattering. Penetration depth of visible region may further decrease during in-vivo imaging due to presence of skin layer; however, this decrease is nominal for NIR regions and therefore skin layer has not been considered in this work. Nevertheless, between NIR-I (740 nm) and NIR-II $(1064 \mathrm{~nm})$, the decrease in scattering is accompanied with an increase in absorption of surrounding tissues, which results in an overall decrease in fluence at higher depths. However, since the maximum skin exposure allowed for NIR-II is higher than NIR-I, the tissue can be irradiated with higher laser energy. Therefore, deep tissue imaging possible at NIR-II can only be attributed to higher illumination energy. Simulation results also showed that absorbance in sphere becomes similar for 800 and $1064 \mathrm{~nm}$ at an approximate depth of $6 \mathrm{~cm}$. Further, absorbance in cylinder and sphere was higher in the NIR-I region than in the NIR-II region, even when illumination was proportional to MPE. Owing to the system restrictions, experimental validation of this trend was not possible as it was difficult to get enough energy density to achieve such imaging depth experimentally.

Another reason of growing popularity of NIR-II window, specifically $1064 \mathrm{~nm}$, is the availability of high laser power at this wavelength. Since traditional Nd:YAG laser can directly deliver pulses of wavelength $1064 \mathrm{~nm}$, there is no need of a dye laser or OPO, which is generally needed for generating wavelengths in NIR-I region. Owing to low efficiency (15\% to $25 \%$ efficiency) of the second-stage laser, their presence not only increases the cost of the system but also results in a decrease in available power. For the PAT experiments illustrated above, maximum laser power of OPO could provide an imaging depth of $25 \mathrm{~mm}$ at 740-nm illumination, whereas maximum laser power of Nd:YAG could provide an imaging depth of $45 \mathrm{~mm}$ at 1064-nm illumination.

In summary, we have compared the PAI depth in breast tissue for illumination of target samples at different wavelengths.
Wavelengths in visible, NIR-I, and NIR-II regions of optical spectrum were compared via MC simulations and via different experiments. We have shown that, for deep tissue imaging, a wavelength of $1064 \mathrm{~nm}$ can be a good option, owing to its high MPE and easy availability of high-power lasers with compact size and cheaper cost. However, as compared to wavelengths in visible region, $\mu_{a}$ of blood is lower at $1064 \mathrm{~nm}$. For this reason, the visible region should be preferred for vasculature imaging at lower depths. Contrast agents are generally needed in NIR region due to low $\mu_{a}$ of blood at those wavelengths. Both blood and ink showed higher absorbance at deeper depths in breast tissue by 1064-nm (NIR-II) illumination compared to 740-nm (NIR-I) illumination at MPE. Therefore, we conclude that for imaging depths $<6 \mathrm{~cm}$ in breast tissue, $1064 \mathrm{~nm}$ (NIR-II) seems more promising than $740 / 800 \mathrm{~nm}$ (NIR-I) for PAI.

\section{Disclosures}

Authors have no relevant financial interest in the paper and no other potential conflicts of interest to disclose.

\section{Acknowledgments}

Srishti would like to thank NTU-India Connect Research Internship Program for providing an opportunity to visit Nanyang Technological University, Singapore. This work was funded by the Singapore Ministry of Health's National Medical Research Council (No. NMRC/OFIRG/0005/2016: M4062012).

\section{References}

1. M. Omar, J. Aguirre, and V. Ntziachristos, "Optoacoustic mesoscopy for biomedicine," Nat. Biomed. Eng. 3, 354-370 (2019).

2. L. Lin et al., "Single-breath-hold photoacoustic computed tomography of the breast," Nat. Commun. 9(1), 2352 (2018).

3. D. Cai et al., "Dual-view photoacoustic microscopy for quantitative cell nuclear imaging," Opt. Lett. 43(20), 4875-4878 (2018).

4. P. K. Upputuri and M. Pramanik, "Recent advances toward preclinical and clinical translation of photoacoustic tomography: a review," $J$. Biomed. Opt. 22(4), 041006 (2017).

5. X. Deán-Ben et al., "Advanced optoacoustic methods for multiscale imaging of in vivo dynamics," Chem. Soc. Rev. 46(8), 2158-2198 (2017).

6. L. V. Wang and J. Yao, "A practical guide to photoacoustic tomography in the life sciences," Nat. Methods 13(8), 627-638 (2016).

7. S. Manohar and D. Razansky, "Photoacoustics: a historical review," Adv. Opt. Photonics 8(4), 586-617 (2016).

8. S. Gottschalk et al., "Rapid volumetric optoacoustic imaging of neural dynamics across the mouse brain," Nat. Biomed. Eng. 3, 392-401 (2019).

9. T. T. Wong et al., "Fast label-free multilayered histology-like imaging of human breast cancer by photoacoustic microscopy," Sci. Adv. 3(5), e1602168 (2017).

10. X. L. Dean-Ben et al., "Volumetric hand-held optoacoustic angiography as a tool for real-time screening of dense breast," J. Biophotonics 9(3), 253-259 (2016).

11. P. J. van den Berg, K. Daoudi, and W. Steenbergen, "Review of photoacoustic flow imaging: its current state and its promises," Photoacoustics 3(3), 89-99 (2015).

12. W. Liu et al., "In vivo corneal neovascularization imaging by opticalresolution photoacoustic microscopy," Photoacoustics 2(2), 81-86 (2014).

13. K. Sivasubramanian, V. Periyasamy, and M. Pramanik, "Non-invasive sentinel lymph node mapping and needle guidance using clinical handheld photoacoustic imaging system in small animal," J. Biophotonics 11(1), e201700061 (2018). 
14. A. Garcia-Uribe et al., "Dual-modality photoacoustic and ultrasound imaging system for noninvasive sentinel lymph node detection in patients with breast cancer," Sci. Rep. 5, 15748 (2015).

15. V. Periyasamy et al., "1064 $\mathrm{nm}$ acoustic resolution photoacoustic microscopy," J. Biophotonics 12(5), e201800357 (2019).

16. K. Sivasubramanian et al., "Hand-held, clinical dual mode ultrasoundphotoacoustic imaging of rat urinary bladder and its applications," J. Biophotonics 11(5), e201700317 (2018).

17. S. Hu and L. V. Wang, "Photoacoustic imaging and characterization of the microvasculature," J. Biomed. Opt. 15(1), 011101 (2010).

18. Y. Han et al., "Three-dimensional optoacoustic reconstruction using fast sparse representation," Opt. Lett. 42(5), 979-982 (2017).

19. S. K. Kalva and M. Pramanik, "Experimental validation of tangential resolution improvement in photoacoustic tomography using a modified delay-and-sum reconstruction algorithm," J. Biomed. Opt. 21(8), 086011 (2016).

20. D. Wang et al., "Coherent-weighted three-dimensional image reconstruction in linear-array-based photoacoustic tomography," Biomed. Opt. Express 7(5), 1957-1965 (2016).

21. L. V. Wang and S. Hu, "Photoacoustic tomography: in vivo imaging from organelles to organs," Science 335(6075), 1458-1462 (2012).

22. X. D. Wang et al., "Noninvasive laser-induced photoacoustic tomography for structural and functional in vivo imaging of the brain," Nat. Biotechnol. 21(7), 803-806 (2003).

23. S. K. Kalva, P. K. Upputuri, and M. Pramanik, "High-speed, low-cost, pulsed-laser-diode-based second-generation desktop photoacoustic tomography system," Opt. Lett. 44(1), 81-84 (2019).

24. W. Xia et al., "Handheld real-time LED-based photoacoustic and ultrasound imaging system for accurate visualization of clinical metal needles and superficial vasculature to guide minimally invasive procedures," Sensors 18(5), 1394 (2018).

25. P. K. Upputuri and M. Pramanik, "Dynamic in vivo imaging of small animal brain using pulsed laser diode-based photoacoustic tomography system," J. Biomed. Opt. 22(9), 090501 (2017).

26. M. Toi et al., "Visualization of tumor-related blood vessels in human breast by photoacoustic imaging system with a hemispherical detector array," Sci. Rep. 7, 41970 (2017).

27. P. K. Upputuri and M. Pramanik, "Photoacoustic imaging in the second near-infrared window: a review," J. Biomed. Opt. 24(4), 040901 (2019).

28. P. K. Upputuri et al., "Contrast-enhanced photoacoustic imaging in the second near-infrared window using semiconducting polymer nanoparticles," J. Biomed. Opt. 24(3), 031002 (2018).

29. G. S. Sangha, E. H. Phillips, and C. J. Goergen, "In vivo photoacoustic lipid imaging in mice using the second near-infrared window," Biomed. Opt. Express 8(2), 736-742 (2017).

30. M. Yang et al., "Photoacoustic/ultrasound dual imaging of human thyroid cancers: an initial clinical study," Biomed. Opt. Express 8(7), 3449-3457 (2017).

31. P. Hai et al., "Near-infrared optical-resolution photoacoustic microscopy," Opt. Lett. 39(17), 5192-5195 (2014).

32. L. A. Sordillo et al., "Deep optical imaging of tissue using the second and third near-infrared spectral windows," J. Biomed. Opt. 19(5), 056004 (2014).

33. American National Standard for Safe Use of Lasers ANSI Z136.1-2007, American National Standards Institute, Inc., New York (2007).

34. E. A. G. Alexey, N. Bashkatov, and V. V. Tuchin, "Tissue optical properties," in Handbook of Biomedical Optics, C. P. D. A. Boas and N. Ramanujan, Eds., pp. 67-100, CRC Press; Taylor and Francis Group, Florida (2011).

35. S. L. Jacques, "Optical properties of biological tissues: a review," Phys. Med. Biol. 58(11), R37-R61 (2013).
36. R. A. Kruger et al., "Photoacoustic angiography of the breast," Med. Phys. 37(11), 6096-6100 (2010).

37. L. V. Wang, S. L. Jacques, and L. Zheng, "MCML-Monte Carlo modeling of light transport in multi-layered tissues," Comput. Methods Programs Biomed. 47(2), 131-146 (1995).

38. V. Periyasamy and M. Pramanik, "Advances in Monte Carlo simulation for light propagation in tissue," IEEE Rev. Biomed. Eng. 10(1), 122-135 (2017).

39. J. G. Koelzer et al., "Measurements of the optical properties of breast tissue using time-resolved transillumination," Proc. SPIE 2326, 143152 (1995).

40. N. Bosschaart et al., "A literature review and novel theoretical approach on the optical properties of whole blood," Lasers Med. Sci. 29(2), 453479 (2014).

41. V. Periyasamy and M. Pramanik, "Monte Carlo simulation of light transport in tissue for optimizing light delivery in photoacoustic imaging of the sentinel lymph node," J. Biomed. Opt. 18(10), 106008 (2013).

42. M. Moothanchery and M. Pramanik, "performance characterization of a switchable acoustic and optical resolution photoacoustic microscopy system," Sensors 17(2), 357 (2017).

43. A. Sharma, S. K. Kalva, and M. Pramanik, "A comparative study of continuous versus stop-and-go scanning in circular scanning photoacoustic tomography," IEEE J. Sel. Top. Quantum Electron. 25(1), $1-9$ (2019).

44. M. Pramanik, "Improving tangential resolution with a modified delayand-sum reconstruction algorithm in photoacoustic and thermoacoustic tomography," J. Opt. Soc. Am. A 31(3), 621-627 (2014).

45. G. Ku et al., "Multiple-bandwidth photoacoustic tomography," Phys. Med. Biol. 49(7), 1329-1338 (2004).

Arunima Sharma received her integrated dual degree with bachelor's degree in bioengineering and master's degree in biomedical technology from the Indian Institute of Technology, Banaras Hindu University, Varanasi, India, in 2016. Currently, she is pursuing her PhD in the School of Chemical and Biomedical Engineering, Nanyang Technological University, Singapore. Her current research interests include development of photoacoustic imaging systems and application of photoacoustics in biomedical imaging.

Srishti was a visiting intern student from the Indian Institute of Technology, Banaras Hindu University, Varanasi, India. She visited Nanyang Technological University, Singapore, through the NTUIndia Connect Research Program. She has a bachelor's degree in bioengineering and a master's in biomedical technology.

Vijitha Periyasamy was a project officer at the School of Chemical and Biomedical Engineering, Nanyang Technological University, Singapore. She has a bachelor's degree in medical electronics from Visvesvaraya Technological University, India. Her research interests include biomedical image processing for clinical evaluation, development of multimodal imaging systems, Monte Carlo (MC) simulation for light transport in biological tissue, and application of bioengineering for different medical practice systems.

Manojit Pramanik received his $\mathrm{PhD}$ in biomedical engineering from Washington University in St. Louis, Missouri, USA. Currently, he is an assistant professor at the School of Chemical and Biomedical Engineering, Nanyang Technological University, Singapore. His research interests include the development of photoacoustic/thermoacoustic imaging systems; image reconstruction methods; clinical application areas such as breast cancer imaging, molecular imaging, and contrast agent development; and MC simulation of light propagation in biological tissue. 